\title{
Flavanone-rich citrus beverages counteract the transient decline in postprandial endothelial function in humans: a randomised, controlled, double-masked, cross-over intervention study
}

\author{
Catarina Rendeiro ${ }^{1} \dagger$, Honglin Dong ${ }^{1} \ddagger$, Caroline Saunders ${ }^{2}$, Laura Harkness ${ }^{3}$, Melvin Blaze $^{4}$, \\ Yanpeng $\mathrm{Hou}^{4}$, Ronald L. Belanger ${ }^{3}$, Giulia Corona ${ }^{1} \S$, Julie A. Lovegrove ${ }^{1}$ and Jeremy P. E. Spencer ${ }^{1 *}$ \\ ${ }^{1}$ Department of Food and Nutritional Sciences, School of Chemistry, Food and Pharmacy, University of Reading, PO Box 226, \\ Reading RG2 6AP, UK \\ ${ }^{2}$ PepsiCo $R+D$ Nutrition, PepsiCo Inc., Reading RG2 6UW, UK \\ ${ }^{3}$ Global R+D Nutrition, PepsiCo Inc., Valhalla, NY 10595, USA \\ ${ }^{4}$ PepsiCo R+D Biological E Discovery Analytics, PepsiCo Inc., New Haven, CT 06511, USA
}

(Submitted 27 June 2016 - Final revision received 23 September 2016 - Accepted 31 October 2016 - First published online 9 January 2017)

\section{Abstract}

Specific flavonoid-rich foods/beverages are reported to exert positive effects on vascular function; however, data relating to effects in the postprandial state are limited. The present study investigated the postprandial, time-dependent $(0-7 \mathrm{~h})$ impact of citrus flavanone intake on vascular function. An acute, randomised, controlled, double-masked, cross-over intervention study was conducted by including middle-aged healthy men (30-65 years, $n$ 28) to assess the impact of flavanone intake (orange juice: $128.9 \mathrm{mg}$; flavanone-rich orange juice: $272 \cdot 1 \mathrm{mg}$; homogenised whole orange: $452.8 \mathrm{mg}$; isoenergetic control: $0 \mathrm{mg}$ flavanones) on postprandial (double meal delivering a total of $81 \mathrm{~g}$ of fat) endothelial function. Endothelial function was assessed by flow-mediated dilatation (FMD) of the brachial artery at $0,2,5$ and $7 \mathrm{~h}$. Plasma levels of naringenin/hesperetin metabolites (sulphates and glucuronides) and nitric oxide species were also measured. All flavanone interventions were effective at attenuating transient impairments in FMD induced by the double meal ( $7 \mathrm{~h}$ post intake; $P<0 \cdot 05)$, but no doseresponse effects were observed. The effects on FMD coincided with the peak of naringenin/hesperetin metabolites in circulation $(7 \mathrm{~h})$ and sustained levels of plasma nitrite. In summary, citrus flavanones are effective at counteracting the negative impact of a sequential double meal on human vascular function, potentially through the actions of flavanone metabolites on nitric oxide.

Key words: Citrus flavanones: Endothelial function: High-fat meals: Nitric oxide: Postprandial state

A transient impairment in vascular function is known to occur in the postprandial or fed state ${ }^{(1-4)}$ and is widely believed to impact on endothelial dysfunction and lifetime CVD risk ${ }^{(5-8)}$. In particular, endothelial function has been shown to be transiently impaired (2-8 h) following ingestion of moderate- to high-fat meals (36-80 g of fat ${ }^{(9-13)}$, potentially driven by hyperglycaemia and hypertriglyceridaemia, which occurs during the postprandial state ${ }^{(1,14,15)}$. Observational data have highlighted that the consumption of diets rich in flavonoids might lead to an improved cardiovascular prognosis $^{(16-20)}$. Indeed, flavonoid-rich foods and beverages are well reported to improve endothelial function acutely in humans both at ${ }^{(21-24)}$ short term ${ }^{(25-27)}$ and long term ${ }^{(28-31)}$. However, most of the acute interventions were undertaken with volunteers in the fasted state, which is considered less representative of the free-living state, whereas data relating to flavonoid potential to ameliorate acutely postprandial endothelial impairments are more scarce $^{(32-34)}$

Although the precise mechanisms by which absorbed flavonoids and their circulating metabolites mediate beneficial vascular effects remain unclear, there is evidence to suggest that the modulation of circulating nitric oxide (NO) levels might be involved ${ }^{(22-24,35-38)}$. Notably, flavanol-rich cocoa has been consistently shown to improve endothelium-dependent vasodilation in healthy individuals ${ }^{(24,27,31,39,40)}$, smokers $^{(23,26)}$, patients with

\footnotetext{
Abbreviations: FMD, flow-mediated dilation; FROJ, flavanone-rich orange juice; NO, nitric oxide; OJ, orange juice beverage; RXNO, nitroso species including nitrosothiols, nitrosamines, iron-nitrosylhaemoglobin and nitrosohaemoglobin; WO, whole orange beverage.

* Corresponding author: J. P. E. Spencer, email j.p.e.spencer@reading.ac.uk

$\dagger$ Present address: Beckman Institute for Advanced Technology, University of Illinois UC, 405 Mathews Av, Urbana, IL 61802, USA.

$\ddagger$ Present address: School of Life Sciences, Faculty of Health and Life Sciences, Coventry University, Coventry, UK.

$\S$ Present address: Life Sciences Department, Whitelands College, University of Roehampton, Holybourne Avenue, London, UK.
} 
coronary artery disease $e^{(41)}$, hypertension ${ }^{(42)}$ or diabetes ${ }^{(43)}$. In particular, acute vascular improvements have been shown to coincide with the appearance of flavanol metabolites in the circulation and with peak plasma NO levels ${ }^{(23,24,26,42)}$. Furthermore, flavanol-induced improvements in vascular function are inhibited following co-administration of an endothelial nitric oxide synthase (eNOS) inhibitor, suggesting a cause-and-effect relationship between flavonoid intake, plasma NO levels and vascular function ${ }^{(23,24)}$. Although less studied, flavanones from citrus fruits have also been shown to exert beneficial effects on human vascular function ${ }^{(28,44)}$. In particular, chronic interventions with orange juice, or the pure flavanone hesperidin, resulted in a decrease in blood pressure in overweight volunteers and acute (6h) improvements in micro-vascular reactivity ${ }^{(45)}$. Short-term intake of pure hesperidin also resulted in significant improvements in endothelial function (as measured by brachial artery flow-mediated dilation (FMD)) in volunteers with the metabolic syndrome $^{(46)}$

In the present study, we assessed the impact of an acute intervention with increasing doses of orange flavanones (sourced from differently processed orange beverages) on human vascular function in the postprandial state. A sequential double meal (breakfast and lunch, delivering a total of $81 \mathrm{~g}$ of fat) was used to simulate the fed state and investigate the postprandial time-dependent effects of flavanone intake on endothelial function as measured by brachial artery FMD.

\section{Methods}

\section{Ethics}

The clinical trial was registered at clinicaltrials.gov (NCT01963416) and conducted according to the Declaration of Helsinki following Good Clinical Practice. It was approved for conduct by the University of Reading's Research Ethical Committee (ethics reference no. 12/06). All volunteers signed an informed consent form before commencing the study.

\section{Intervention study volunteers}

Volunteers were recruited from the University of Reading and surrounding areas using the Hugh Sinclair Unit volunteers' database, poster advertisements within the university and local community via local websites (April-December 2012). In total, fifty-nine, healthy, male volunteers, aged 30-65 years, were assessed for screening and were selected according to the following inclusion criteria: (1) fasting lipids in the upper half of the normal range (TAG $0.8-3.2 \mathrm{mmol} / 1$ and total cholesterol $6.0-8.0 \mathrm{mmol} / \mathrm{l})$ or BMI $25-32 \mathrm{~kg} / \mathrm{m}^{2}$; (2) non-smoker; (3) non-diabetic (diagnosed or fasting glucose $>7 \mathrm{mmol} / \mathrm{l}$ ) with no endocrine disorders; (4) $\mathrm{Hb}$ and liver enzyme levels within the normal range; (5) not having suffered a myocardial infarction/ stroke in the past 12 months; (6) not suffering from renal or bowel diseases or having a history of cholestatic liver or pancreatitis; (7) not on drug treatment for hyperlipidaemia, hypertension, inflammation or hyper-coagulation; (8) not taking any fish oil, fatty acid or vitamin and mineral supplements; (9) no history of alcohol misuse; (10) not planning weight loss or on a weight-reducing regimen; (11) not taken antibiotics during the 6 months before the study; and (12) not being able to consume the study meals. Those selected for the study were instructed not to alter their usual dietary or fluid intakes. Volunteers were asked to refrain from the following for $24 \mathrm{~h}$ before and during the study: (1) consumption of polyphenol-rich foods including fruits (including citrus fruits), vegetables, cocoa, chocolate, coffee, tea, fruit juices and wine; (2) consumption of foods rich in nitrates, including beetroot, spinach, lettuce, rocket, celery, parsley and cabbage (defined as containing $>50 \mathrm{mg}$ nitrates $/ 100 \mathrm{~g}$ fresh weight $^{(47)}$; (3) performing rigorous exercises; and (4) consuming any alcoholic beverage. Volunteers were further asked to fast for $12 \mathrm{~h}$ before each study visit, and during that period they were to only consume the low-nitrate water provided. The same standard meal, low in polyphenols and nitrates, was also provided for dinner for the day before each visit. Written informed consent was obtained from all eligible volunteers before their participation in the study.

\section{Study design}

The study design was an acute, randomised, placebo-controlled, double-masked, postprandial, cross-over study (Fig. 1). After the initial screening visit to assess the eligibility of volunteers for participation, volunteers were enrolled into the study (by researchers C. R. and H. D.) and visited the Hugh Sinclair Unit at the University of Reading on four separate occasions separated by a 2-week period (June-December 2012). Volunteers were asked to consume either a (a) control drink (C), (b) orange juice beverage (OJ), (c) flavanone-rich orange juice (FROJ) or (d) whole orange beverage (WO), together with a high-fat breakfast (at baseline, $t=0 \mathrm{~h}$ ), followed by a medium-fat lunch $(t=5.5 \mathrm{~h})$. H. D. assigned participants to the three-digit coded drink interventions for their four visits according to a random allocation sequence generated by a third party. Details on flavonoid composition of the interventions, as well as micronutrient and macronutrient compositions, can be found on Table 1. Compliance to a 24-h low-polyphenol intake period and $12 \mathrm{~h}$ of fasting was monitored using a 24-h dietary recall conducted
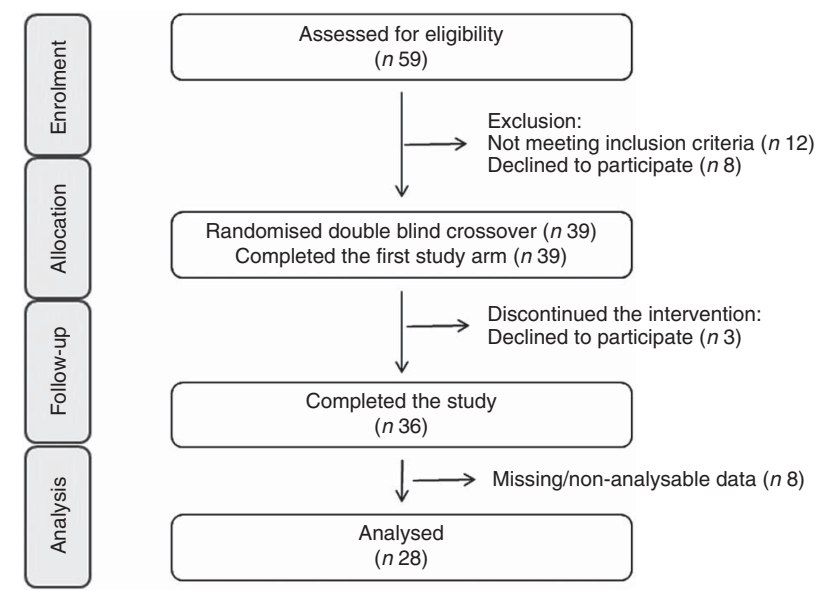

Fig. 1. Consolidated Standards of Reporting Trials flow diagram for the postprandial study. 
during each study visit. On each visit day, volunteers rested for $30 \mathrm{~min}$ in a quiet, temperature-controlled room before they were cannulated by a qualified research nurse; blood samples were collected in the fasted state $(0 \mathrm{~h})$ and at 2, 5, 7 and $24 \mathrm{~h}$ after consumption of each intervention drink. FMD of the brachial artery was the primary outcome and it was measured at $0,2,5$ and $7 \mathrm{~h}$ after consumption. Secondary outcomes of the study included systolic and diastolic blood pressures (0, 2, 5, 7h), plasma flavanone levels $(0,2,5,7,24 \mathrm{~h})$ and NO plasma levels $(0,2,5,7 \mathrm{~h})$. After baseline measurements were obtained, the high-fat breakfast (Table 2) was consumed with one of the clinical products (C, OJ, FROJ, WO). Volunteers were asked to consume the intervention drink and the high-fat meal within $10-15 \mathrm{~min}$. At $5.5 \mathrm{~h}$ from baseline, a medium-fat lunch was provided (Table 2). The last measurement of the day was performed at $7 \mathrm{~h}$, and the volunteers were asked to return to the clinical unit the following morning to provide a $24 \mathrm{~h}$ h blood sample (fasted). From 7 to $24 \mathrm{~h}$, volunteers were asked to consume the free polyphenol dinner provided by the research team and to continue on the low-polyphenol diet, as well as refrain from exercise and alcohol consumption. Blood samples for flavonoid analysis were collected in EDTA-containing tubes (Greiner Bio-One Ltd), immediately centrifuged for $15 \mathrm{~min}$ at $4^{\circ} \mathrm{C}(4000 \mathrm{~g})$, and the plasma samples were spiked with formic acid $(1.5 \%$ of a $50 \%$ water

Table 1. Compositional analysis of orange flavanone beverages and control beverage used in the acute postprandial study

(2 Relative standard deviation of the measurement (2-RSD))

\begin{tabular}{|c|c|c|c|c|c|}
\hline \multirow[b]{2}{*}{ Compounds } & \multirow[b]{2}{*}{ 2-RSD (\%) } & \multicolumn{4}{|c|}{ Intervention drink (240 ml) } \\
\hline & & Control & OJ & FROJ & WO \\
\hline Hesperidin (mg) & $5 \cdot 0$ & - & $107 \cdot 30$ & $220 \cdot 46$ & 352.80 \\
\hline Narirutin (mg) & $5 \cdot 0$ & 0.08 & $15 \cdot 41$ & 34.54 & $76 \cdot 58$ \\
\hline Others $(\mathrm{mg})^{\star}$ & & 0.02 & $6 \cdot 17$ & $17 \cdot 14$ & 23.33 \\
\hline Total flavonoids (mg) & & 0.10 & $128 \cdot 88$ & $272 \cdot 14$ & 452.71 \\
\hline Fructose $(\mathrm{g})$ & 4.0 & $6 \cdot 38$ & 6.63 & $6 \cdot 12$ & 6.89 \\
\hline Glucose (g) & 4.0 & $5 \cdot 36$ & $5 \cdot 36$ & $5 \cdot 10$ & $5 \cdot 87$ \\
\hline Sucrose $(\mathrm{g})$ & $2 \cdot 0$ & $10 \cdot 20$ & $10 \cdot 97$ & 11.99 & 11.48 \\
\hline Total sugars (g) & & 21.93 & 22.95 & 23.21 & 24.23 \\
\hline Fibre (total) (g) & $12 \cdot 0$ & - & 0.66 & $5 \cdot 36$ & $6 \cdot 30$ \\
\hline Ascorbic acid (mg) & 8.0 & - & $105 \cdot 57$ & $80 \cdot 17$ & $123 \cdot 01$ \\
\hline Folate $(\mu \mathrm{g})$ & $16 \cdot 0$ & - & 54.06 & $65 \cdot 28$ & 64.77 \\
\hline Total $\beta$-carotenes (mg) & $15 \cdot 0$ & - & 0.13 & 0.26 & 0.35 \\
\hline
\end{tabular}

Control, sugar matched control; OJ, Tropicana pure premium orange juice without pulp; FROJ, flavanone-rich orange juice: Tropicana pure premium orange juice with added orange pomace; WO, juice made from lightly blended fresh whole orange. * Includes diosmin, didymin, nobiletin, tangeretin, sinensetin, Me4-scutellarein.

Table 2. Macronutrient composition of double-meal protocol solution) and ascorbic acid ( $5 \%$ of a $10 \mathrm{~mm}$ solution) and stored at $-80^{\circ} \mathrm{C}$. Blood samples for NO analysis were collected in heparincontaining tubes, immediately (within $3 \mathrm{~min}$ of collection) centrifuged for $15 \mathrm{~min}$ at $4^{\circ} \mathrm{C}(4000 \mathrm{~g})$, and plasma was rapidly collected, aliquoted and stored at $-80^{\circ} \mathrm{C}$ to reduce inactivation of nitroso species.

\section{Sequential double meal}

The sequential double-meal protocol was based on the department's extensive experience on postprandial studies, which have been collated into the Dietary Studies: Reading Unilever Postprandial Trials (DISRUPT) database ${ }^{(48)}$. It consisted of two meals: (1) high-fat breakfast ( $51 \mathrm{~g}$ fat; $14 \mathrm{~g}$ protein; $64 \mathrm{~g}$ carbohydrates; $3251 \mathrm{~kJ}$ (777 kcal)) administered with the intervention drink and (2) medium-fat lunch (30 g fat; $15 \mathrm{~g}$ protein; $80 \mathrm{~g}$ carbohydrates; $2628 \mathrm{~kJ}$ (628 kcal)) (Table 2) administered $5.5 \mathrm{~h}$ after the intervention drink. The high-fat meal consisted of two butter croissants ( $47 \mathrm{~g}$ of fat) and $5 \mathrm{~g}$ of butter ( $4 \mathrm{~g}$ of fat). The medium-fat meal consisted of two slices of white bread ( $2 \mathrm{~g}$ of fat), $42 \mathrm{~g}$ of Philadelphia soft cheese (13 $\mathrm{g}$ of fat), a small bag of salted crisps ( $9 \mathrm{~g}$ of fat) and two shortbread biscuits ( $6 \mathrm{~g}$ of fat) (Table 2 ). The volunteers were asked to consume meals within 10-15 min.

\section{Flavanone-containing interventions}

The preparations of the intervention drinks were carried out according to good manufacturing practice as described in hazard analysis and critical control points (HACCP). The control (C) drink was matched for sugars found in the orange beverages, and $0.67 \%$ citric acid and orange flavouring were added to enhance flavour. The levels of total $\beta$-carotenes present in the flavanone treatments were considered negligible (approximately $0.25 \mathrm{mg} ; 2$ relative standard deviation of the measurement (2-RSD): $15 \%$ ) with regard to endothelial function effects, with a 15-mg dose (6 weeks intervention, in combination with $150 \mathrm{mg}$ of vitamin C) resulting in no significant changes on endothelial biomarkers ${ }^{(49)}$ (Table 1). The levels of folate present (approximately $60 \mu \mathrm{g} ; 2$-RSD: 16\%) can also be considered insignificant with regard to its potential to impact on endothelial function; folate has been shown to drive small improvements in endothelial function only in long-term interventions (1-4 months) with at least 5000-10 000 $\mu \mathrm{g} / \mathrm{d}$ of folate, but not with lower doses in the ranges of $400-800 \mu \mathrm{g} / \mathrm{d}^{(50)}$.

\begin{tabular}{|c|c|c|c|c|c|c|}
\hline & \multirow[b]{2}{*}{ Foods } & \multicolumn{3}{|c|}{ Macronutrient breakdown } & \multirow[b]{2}{*}{ Energy (kJ) } & \multirow[b]{2}{*}{ Energy (kcal) } \\
\hline & & Fat $(\mathrm{g})$ & Protein (g) & Carbohydrates (g) & & \\
\hline \multirow[t]{3}{*}{ High-fat breakfast } & Butter croissant $(2 x)$ & 47 & 14 & 64 & 3096 & 740 \\
\hline & Butter $(5 \mathrm{~g})$ & 4 & N/A & $\mathrm{N} / \mathrm{A}$ & 159 & 37 \\
\hline & Total & 51 & 14 & 64 & 3251 & 777 \\
\hline \multirow[t]{5}{*}{ Medium-fat lunch } & 2 slices of sliced white bread $(108 \mathrm{~g})$ & 2 & 8.5 & 50 & 992 & 237 \\
\hline & Philadelphia soft cheese $(42 \mathrm{~g})$ & 13 & 3.6 & $\mathrm{~N} / \mathrm{A}$ & 548 & 131 \\
\hline & Crisps $(25 \mathrm{~g})$ & 9 & 1.5 & 13 & 556 & 133 \\
\hline & Shortbread biscuit (22 g) & 6 & 1.4 & 16 & 531 & 127 \\
\hline & Total & 30 & 15 & 80 & 2628 & 628 \\
\hline
\end{tabular}


The OJ used for the intervention was a commercial $100 \%$ pure orange juice (Tropicana Pure Premium). The FROJ used was Tropicana Pure Premium with added orange pomace. Pomace is the edible part of a whole orange that is leftover during the production of Tropicana pure premium orange juice and subjected to particle size reduction. Orange pomace is rich in fibre (40:60 ratio of soluble:insoluble) and contains small amounts of micronutrients and a high proportion of the polyphenols found in whole orange. The WO consisted of lightly blended whole table orange, without the peel. Drinks displayed slightly different viscosities, but specific measurements were not undertaken to assess this. All drinks were stored in individual portions $(255 \mathrm{~g} / 240 \mathrm{ml})$ in aluminium canisters, frozen at $-20^{\circ} \mathrm{C}$ and labelled with a three-digit code to ensure double-masking. Drinks were defrosted overnight in the fridge $\left(4^{\circ} \mathrm{C}\right)$ just before being used on each study day. Participants, care-providers and all researchers assessing outcomes were blinded until all the data were analysed. Quantification of flavanones from orange beverages (OJ, FROJ and WO) was performed by ultra-HPLC coupled with tandem MS (UHPLC-MS/MS). Sample preparation was performed by diluting the juice sample with dimethyl sulfoxide, the internal standard (IS) solution $(10 \mu \mathrm{g} / \mathrm{ml}$ d4-dimethyl phthalate in 50\% acetonitrile-water) and 50\% acetonitrile-water, followed by vortexing and centrifugation $(10 \mathrm{~min}$, $2500 \mathrm{rpm}$ ). The supernatant was filtered before analysis in an Agilent 1290 UHPLC (Agilent Technologies), with a Zorbax Eclipse Plus C18 column $(1.8 \mu \mathrm{m}, 2.1 \times 100 \mathrm{~mm}$; linear gradient starting at $100 \%$ (A) containing $2 \%$ acetonitrile in water with $0.1 \%$ formic acid, to $90 \%$ (B) containing acetonitrile with $0.1 \%$ formic acid, followed by $100 \%$ B). MS detection was performed in electrospray ionisation (ESI)-positive ion mode on an Agilent 6530A Q-ToF MS with MassHunter Software for instrument control and data processing. Calibration standards were prepared from analytical grade materials purchased from Indofine Chemical Chromadex or LKT Laboratories. The levels of flavanones in the test products are presented in Table 1. In brief, the total levels of flavanones in (a) OJ was $128.88 \mathrm{mg}$, (b) in FROJ $272.14 \mathrm{mg}$ and (c) in WO $452.80 \mathrm{mg}$ (Table 1). The flavanone hesperidin was the main flavonoid present in the intervention beverages, ranging from $107.30 \mathrm{mg}$ (OJ) to $352 \cdot 80 \mathrm{mg}$ (WO).

\section{Flow-mediated dilation}

FMD of the brachial artery was the primary end point measure of the study, and measurements were taken following standard guidelines $^{(51)}$ using an ALT Ultrasound HDI5000 system (ATL Ultrasound) in combination with a semi-automated computerised analysis system (Brachial Analyzer; Medical Imaging Applications LLC). In brief, after $15 \mathrm{~min}$ of rest in the supine position in a quiet air-conditioned room, the brachial artery was imaged longitudinally at $2-10 \mathrm{~cm}$ proximal to the antecubital fossa. After baseline images were recorded for $60 \mathrm{~s}$, a blood pressure cuff placed around the forearm was inflated to $220 \mathrm{mmHg}$. After $5 \mathrm{~min}$ of occlusion, the pressure was rapidly released to allow reactive hyperaemia, with image collection continuing for $5 \mathrm{~min}$ after release. A single researcher, who was blinded to the measurement details, analysed all image files, and peak diameter was defined as the largest diameter obtained after the occlusion was released. FMD response was calculated as relative diastolic diameter change from baseline as compared with peak diastolic diameter. A total of twenty-eight volunteers were analysed for their FMD response. Data from eight volunteers were not analysed or were excluded because of the following reasons: (i) measurement of FMD from the non-dominant arm (rather than dominant) due to limitations with blood collection ( $n$ 2); (ii) absence of FMD response ( $n$ 3); and (iii) technical problems during recording of ultrasound FMD measurements rendered non-analysable data ( $n$ 3).

\section{Blood pressure}

Systolic and diastolic blood pressures were measured using an Omron MX2 automatic digital upper-arm blood pressure monitor (Omron Healthcare UK Ltd). All measurements were taken according to standard practice and by a qualified research nurse, before and following each intervention period. Before starting blood pressure measurements, the volunteers were seated or were laying down quietly for at least $20 \mathrm{~min}$. Measurements were taken in the right arm, before FMD procedure for each time point. The subject's right arm was allowed to rest on a pillow (on a side table positioned at heart level) and was slightly flexed with the palm facing upward. Volunteers were asked to refrain from speaking during blood pressure measurements. The measurements were repeated three times, and blood pressure was considered as the average of these measurements.

\section{Plasma flavanone analysis}

Blood samples were collected in EDTA-containing tubes and centrifuged at $4^{\circ} \mathrm{C}$ for $10-15 \mathrm{~min}$ at $4000 \mathrm{~g}$. Formic acid (1.5\% of a $50 \%$ solution) and ascorbic acid (5\% of a 10-mm solution prepared fresh everyday) were added to the plasma samples to preserve flavanones before freezing at $-80^{\circ} \mathrm{C}$. A subset of twenty volunteers was selected randomly for analysis of flavanone content. A high throughput analytical method using UHPLC-MS/MS was developed and validated to measure simultaneously naringenin and hesperetin in human plasma. Enzymatic hydrolysis and methanol extraction were applied as described earlier ${ }^{(52)}$ with modifications to accommodate in situ monitoring of enzyme efficiency and automated sample preparation using a Hamilton Microlab Star liquid handling system (Hamilton). Plasma samples ( 45 litres) were incubated after addition of $\beta$-glucuronidase type VIIA (Sigma) and sulfatase type H-5 (Sigma) for 90 and $60 \mathrm{~min}$ at $37^{\circ} \mathrm{C}$, respectively. To monitor enzyme activity, every individual sample was spiked with a known concentration of phenolphthalein glucuronide and potassium 4-nitrophenyl sulfate (Sigma) as enzyme substrates in addition to caffeine-(trimethyl-d9) (Sigma) as IS before incubation. The enzyme-hydrolysed samples were subsequently extracted with methanol and centrifuged. The supernatant (6 litres) was analysed using an Agilent 1290 UHPLC coupled with an Agilent 6490 triple quadrupole MS (Agilent Technologies). Naringenin and hesperetin were separated in a Waters BEH C18 $(100 \times 2.1 \mathrm{~mm}, 1.7$ micrometre particle size $)$ at a flow rate of $0.6 \mathrm{ml} /$ min using 6.5-min gradient $99 \%$ solvent A (water containing $0 \cdot 1 \%$ formic acid) and $1 \%$ solvent $B$ (acetonitrile containing $0.1 \%$ formic acid) (initially; $70 \%$ solvent $\mathrm{A}$ at $0.5 \mathrm{~min} ; 55 \%$ solvent $\mathrm{A}$ at $2.5 \mathrm{~min}$; $2 \%$ solvent A at $3.0 \mathrm{~min} ; 2 \%$ solvent A at $4.0 \mathrm{~min} ; 99 \%$ solvent A at $4.5 \mathrm{~min}$ followed by post-equilibration for $2 \mathrm{~min}$ ). The MS was 
operated in ESI-positive ionisation mode and multiple reaction monitoring (MRM) mode by monitoring quantifier and qualifier ions for both naringenin and hesperetin. MRM transitions were determined as 204.1/144.0 $(\mathrm{m} / \mathrm{z})$ corresponding to caffeine, as $495 \cdot 1 / 319 \cdot 1(\mathrm{~m} / z)$ corresponding to phenolphthalein-glucuronide and as $217.9 / 137.9(\mathrm{~m} / \mathrm{z})$ corresponding to potassium 4-nitrophenyl sulphate as quantifier ions. MRM transitions were determined as $303 \cdot 1 / 153 \cdot 1(\mathrm{~m} / z)$ corresponding to hesperetin and $273 \cdot 1 / 147 \cdot 1(\mathrm{~m} / \mathrm{z})$ corresponding to naringenin as qualifier ions. Concentrations of hesperetin and naringenin were then calculated on the basis of ratios of their integrated peak area for the quantifier ions to that of IS using two sets of eight-point calibration curves. Accuracy of the analysis was monitored by systematic counterbalancing between plasma samples and quality control samples spiked with known concentrations of hesperetin and naringenin. The method was validated for a linear calibration range of $0.0313-8.02 \mu \mathrm{m}$ for naringenin and $0.0282-7.22 \mu \mathrm{m}$ for hesperetin, respectively. In addition, limits of detection for naringenin and hesperetin were determined as 2 and $7 \mathrm{~nm}$, respectively.

\section{Biochemical analysis}

The blood samples collected in pre-chilled Li or heparin tubes were spun ( $4000 \mathrm{~g} ; 10-15 \mathrm{~min} ; 4^{\circ} \mathrm{C}$ ) immediately after collection (within $3 \mathrm{~min}$ ). Samples were also collected in serum separation tubes and allowed to stand for $30 \mathrm{~min}$ before centrifugation ( $1300 \mathrm{~g} ; 10 \mathrm{~min} ; 21^{\circ} \mathrm{C}$ ). All samples were aliquoted and frozen at $-80^{\circ} \mathrm{C}$ until analysis.

Plasma nitric oxide analysis. Plasma samples for measurement of total nitroso species (NOx) were aliquoted in 150- $\mu$ l aliquots to avoid freeze-thawing of the samples for each measurement. Samples were defrosted just before the measurements took place (within $10 \mathrm{~min}$ ) and were kept on ice throughout. Plasma samples ( $n$ 28) were analysed for nitrite, nitrate and other nitroso species (RXNO, including nitrosothiols, nitrosamines, ironnitrosylhaemoglobin and nitrosohaemoglobin) by ozone-based chemiluminescence (model 88AM; Eco Physics) as previously described $^{(53)}$. In brief, for total NOx measurement (NO derived from nitrate, nitrite and RXNO), one aliquot of plasma was injected into an airtight microreaction vessel containing a solution of vanadium (III) chloride ( $50 \mathrm{~mm}$ ) dissolved in $1 \mathrm{~m}-\mathrm{HCl}$, connected to a chemiluminescence analyser. For measurement of nitrite and other RXNO (i) one aliquot of plasma was injected in the same apparatus into glacial acid acetic solution containing $45 \mathrm{~mm}$-potassium iodide and $10 \mathrm{~mm}$-iodide, at $60^{\circ} \mathrm{C}$, actively purged by inert $\mathrm{He}$, which allowed the detection of $\mathrm{NO}$ from both nitrite and RXNO (but no nitrate). (ii) Subsequently, the plasma sample was treated with acidic sulphanilamide $(1 \mathrm{~m}-\mathrm{HCl})$ to scavenge nitrite, before injection, allowing for quantification of RNXO alone. Nitrite levels in the plasma samples were determined by the difference between these two measurements (i and ii). Nitrate concentration was determined by subtracting nitrite + RXNO from total NOx. Samples used for calibration curves were prepared fresh every day and displayed consistent values across days.

Plasma baseline lipids and glucose. Plasma levels of total cholesterol, LDL-cholesterol, HDL-cholesterol, glucose and TAG were assayed on an ILAB 600 chemistry analyzer (Instrumentation Laboratory) using enzyme-based colourimetric tests supplied by Instrumentation Laboratory.

\section{Power calculation and statistical analysis}

Power calculations were performed for the primary end point change in FMD response. Power was based on the intra-individual variability of the operator who performed the FMD analysis $(5 \% \mathrm{CV}, \mathrm{SD}=0.3 \%)$. Previous measures of variability in a control group estimated the standard deviation within subjects to be $2.3 \%$. At $90 \%$ power and 0.05 significance, the number of volunteers required to detect a difference of $1.5 \%$ in the response of matched pairs in a cross-over study was twenty-five. All statistical analyses were performed using SPSS Statistics 21 (IBM) package. FMD, blood pressure, plasma levels of nitric oxide species (nitrate, nitrite and RNXO) and plasma levels of flavanones were analysed using a two-way repeated measures ANOVA within subjects with time $(0,2,5,7 \mathrm{~h})$ and treatment $(\mathrm{C}, \mathrm{OJ}, \mathrm{FROJ}, \mathrm{WO})$ as main factors. Post hoc and pair-wise comparisons were carried out using the Bonferroni correction for multiple comparisons. Significance was defined as $P<0.05$ (95\% CI) for all outcome measures, with $P$ values represented in the figures as follows: $* P=0 \cdot 01-0 \cdot 05$, $* P=0.001-0.01, * * P<0.001$. Pharmacokinetic parameters were calculated as follows: (a) the maximum plasma concentration $\left(C_{\max }\right)$ and (b) the time to reach the maximum plasma concentration $\left(T_{\max }\right)$ were determined from the individual data obtained from each participant; (c) the area under the plasma concentration $v$, time curve (AUC) was calculated using the trapezoidal method. Multiple regression analysis was used to predict the value of FMD (dependent variable) based on the values of hesperetin and naringenin plasma levels (independent variables). Random allocation sequence was generated by a third party statistician using SAS version 9.1 (procedure plan and seed $=122700$ ). The randomised block design contained four blocks and nine randomised sequences within each block.

\section{Results}

\section{Baseline characteristics and tolerance of intervention}

The baseline characteristics of volunteers recruited were within the desired ranges, with TAG ranging from 0.8 to $3.2 \mathrm{mmol} / \mathrm{l}$ and total cholesterol from 6.0 to $8.0 \mathrm{mmol} / \mathrm{l}$ or/and BMI from 25 to $32 \mathrm{~kg} / \mathrm{m}^{2}$ (Table 3). All intervention beverages were well tolerated by all volunteers, as well as the high- and medium-fat meals administered throughout the study. No adverse events were reported.

\section{Flavanone modulation of postprandial flow-mediated dilatation}

A two-factor repeated-measures ANOVA for endotheliumdependent brachial artery vasodilation, measured FMD response, revealed a highly significant interaction between the interventions (C, OJ, FROJ, WO $)$ and time of the day $(0,2,5,7 \mathrm{~h})\left(F_{9,243}=3 \cdot 27\right.$, $P<0.0001)$, as well as significant main effects of time of the day $\left(F_{3,81}=12.062, P<0.0001\right)$ and intervention $\left(F_{3,81}=2.78, P<0.05\right)$. At baseline $(t=0 \mathrm{~h})$, there were no significant differences in 
Table 3. Baseline clinical characteristics of the study population (Mean values with their standard errors)

\begin{tabular}{|c|c|c|}
\hline Baseline characteristics & Mean & SEM \\
\hline Age (years) & 48 & 1 \\
\hline BMI $\left(\mathrm{kg} / \mathrm{m}^{2}\right)$ & 28.4 & 0.4 \\
\hline Total cholesterol $(\mathrm{mmol} / \mathrm{l})$ & $5 \cdot 6$ & 0.2 \\
\hline HDL-cholesterol (mmol/l) & 1.3 & 0.3 \\
\hline TAG $(\mathrm{mmol} / \mathrm{l})$ & 1.5 & 0.1 \\
\hline Fasting glucose $(\mathrm{mmol} / \mathrm{l})$ & 4.8 & 0.1 \\
\hline $\mathrm{Hb}(\mathrm{g} / \mathrm{l})$ & 149 & 1.0 \\
\hline Systolic blood pressure $(\mathrm{mmHg})$ & 124.0 & 1.7 \\
\hline Diastolic blood pressure $(\mathrm{mmHg})$ & 74.9 & 1.5 \\
\hline \multicolumn{3}{|l|}{ Liver enzymes } \\
\hline Alanine aminotransferase (IU/I) & $42 \cdot 1$ & 2.1 \\
\hline$y$-Glutamyltransferase (IU/I) & 41.7 & $5 \cdot 3$ \\
\hline
\end{tabular}

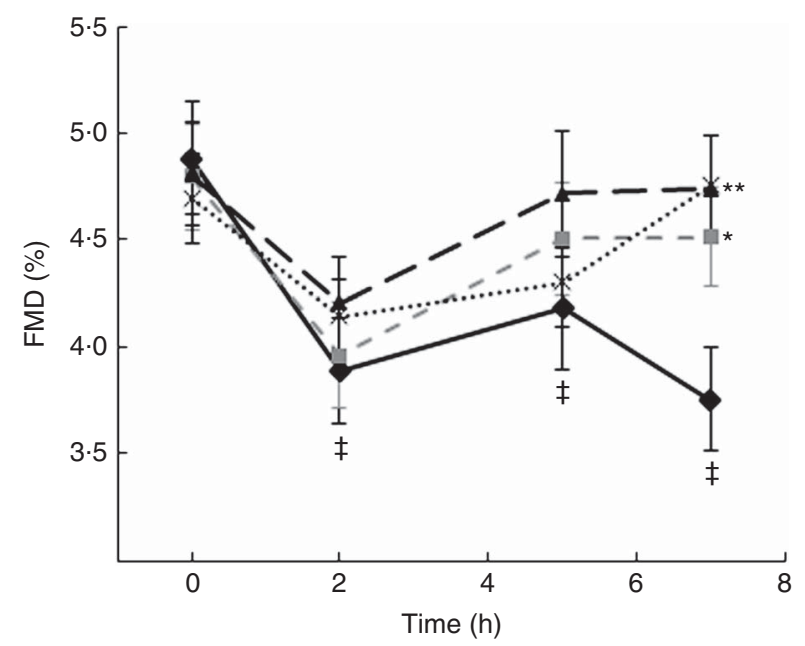

Fig. 2. Time course of postprandial flow-mediated dilatation (FMD) following consumption of flavanone beverages containing either $128.88 \mathrm{mg}$ of flavanones (OJ, - - ), $272.14 \mathrm{mg}$ of flavanones (FROJ, - - ), $452.80 \mathrm{mg}$ of flavanones (WO, .......) or a macronutrient- and micronutrient-matched control ( - ) in middle-aged healthy men ( $n$ 28). A high-fat breakfast $(51 \mathrm{~g}$ of fat) was administered at $t=0 \mathrm{~h}$, and a medium-fat lunch ( $30 \mathrm{~g}$ of fat) was administered at $t=5.5 \mathrm{~h}$. Values are means, with their standard errors and analysed using a two-factor repeated-measures ANOVA with time and treatment as the two factors (significant main effects of time $\times$ treatment $(P<0.0001)$, time $(P<0.0001)$ and treatment $(P<0.05))$. Post hoc analyses were conducted using Bonferroni's multiple comparison test. * $P<0.05$ OJ significantly different from control at the $7 \mathrm{~h} ;{ }^{\star *} P<0.01 \mathrm{FROJ}$ and WO significantly different from control at $7 \mathrm{~h}$. $\ddagger$ Significant decrease in FMD response in relation to baseline levels for both control (at 2, 5 and $7 \mathrm{~h} ; P<0.0001, P<0.05, P<0.0001$, respectively) and all three flavanone interventions (at $2 \mathrm{~h} ; P<0.05$ ). OJ, orange juice; FROJ, flavanone-rich orange juice; WO, whole blended orange.

brachial artery FMD between visits, with the average baseline levels of FMD for the study population being 4.80 (SEM 0.03) FMD units. After $2 \mathrm{~h}$ of intake of the high-fat meal, a significant decrease in \% FMD was detected for both control $(P<0 \cdot 0001)$ and the three flavanone interventions $(P<0.05)$ (Fig. 2). In particular, in the control group, the \% FMD decreased by 0.99 (SEM 0.17)\% FMD after $2 \mathrm{~h} \quad(P<0.0001)$ and remained significantly suppressed $5 \mathrm{~h}(P<0.05)$ and $7 \mathrm{~h}(P<0.0001)$ after intake, relative to baseline levels. In contrast, all orange flavanone interventions resulted in a recovery in \% FMD to that of baseline levels between 5 and $7 \mathrm{~h}$ (OJ: 4.51 (SEM 0.23)\% FMD; FROJ: 4.74 (SEM 0.25 )\%
FMD and WO: 4.75 (SEM 0.23)\% FMD) (Fig. 2). At $5 \mathrm{~h}$ post intervention, there were no significant differences in \% FMD between control and each of the flavanone interventions (OJ, FROJ, WO), whereas at $7 \mathrm{~h}$ (following intake of the medium-fat meal at $5.5 \mathrm{~h}$ ) we observed a significantly higher \% FMD for OJ $(P<0.05)$, FROJ $(P<0.01)$ and WO $(P<0.01)$ in comparison with control. There were no significant differences between the flavanone interventions at $7 \mathrm{~h}$, with all three doses of flavanones administered (OJ: $128.8 \mathrm{mg}$; FROJ: $272.1 \mathrm{mg}$ and WO: $452.7 \mathrm{mg}$ ) counteracting the deleterious effect of the double-meal challenge on \% FMD response to a similar extent. Blood pressure was not significantly altered following consumption of any of the flavanone interventions, relative to baseline or to the control beverage (Table 4).

\section{Modulation of plasma flavanones}

Total flavanones, naringenin and hesperetin (including glucuronides and sulphates), were not detected in the plasma of volunteers at baseline, indicating compliance to the 24-h low-flavonoid diet before the study visits. Flavanone metabolites were not detected in the circulation of individuals following intake of the control drink (Fig. 3). Significant increases in plasma levels of hesperetin metabolites were detected at 5 and $7 \mathrm{~h}(P<0.0001)$ (Fig. 3(a)) and at 2, 5 and $7 \mathrm{~h}$ for naringenin metabolites $(P<0 \cdot 0001)$ (Fig. 3(b)). The time to reach $T_{\max }$ for hesperetin and naringenin was not significantly different between treatments and coincided with the timeframe of FMD effects (Table 5). At 2, 5 and $7 \mathrm{~h}$, plasma levels of naringenin were significantly higher following WO intake compared with OJ $(P<0 \cdot 01)$ (Fig. 3(b)). Similarly, at $7 \mathrm{~h}$, both FROJ and WO showed a trend towards higher plasma concentrations of hesperetin, relative to OJ $(P<0 \cdot 1)$ (Fig. 3(a)). With respect to the $C_{\max }$ and AUC $(0-24 \mathrm{~h})$ for plasma hesperetin, both FROJ and WO were significantly higher than after OJ $(P<0.05)$, whereas for naringenin, both $C_{\max }$ and AUC were only significantly higher for WO in relation to OJ $(P<0.005)$ (Table 5). No significant differences in plasma flavanone levels were detected between FROJ and WO despite the different levels present in the treatment drinks. At $24 \mathrm{~h}$, the levels of flavanones were not significantly different from baseline, indicating that the flavanone metabolites have been cleared from circulation $(P=0 \cdot 13)$ (Fig. 3). A multivariate regression analysis, including both plasma naringenin and hesperetin, showed that hesperetin $(P=0.001)$, but not naringenin $(P=0.092)$, predicted changes in \% FMD over the course of $0-7 \mathrm{~h}$. Specifically, at $7 \mathrm{~h}$, at the peak of FMD response, hesperetin significantly predicted the magnitude of FMD increase $(r 0.32, P=0.005)$ following flavanone intake.

\section{Modulation of plasma nitrite, nitrate and nitroso species including nitrosothiols, nitrosamines, iron-nitrosylhaemoglobin and nitrosohaemog/obin}

Levels of nitrate, nitrite and other RXNO species were determined in plasma at baseline and 2, 5 and $7 \mathrm{~h}$ after treatment (Fig. 4). Nitrite plasma levels are known to reflect more 
Table 4. Acute postprandial effects of orange flavanone beverages on static blood pressure

\begin{tabular}{|c|c|c|c|c|c|c|c|c|c|}
\hline Blood pressure $(\mathrm{mmHg})$ & \multicolumn{2}{|c|}{ Baseline } & \multicolumn{2}{|c|}{$2 \mathrm{~h}$} & \multicolumn{2}{|c|}{$5 \mathrm{~h}$} & \multicolumn{2}{|c|}{$7 \mathrm{~h}$} & $P$ \\
\hline \multicolumn{10}{|l|}{ Systolic } \\
\hline Control & 125.5 & 1.9 & $126 \cdot 7$ & 1.9 & $126 \cdot 7$ & 1.7 & $128 \cdot 6$ & 1.5 & NS \\
\hline OJ & 124.8 & 1.5 & $125 \cdot 7$ & $1 \cdot 2$ & $125 \cdot 72$ & 1.5 & $126 \cdot 4$ & 1.5 & \\
\hline FROJ & $126 \cdot 1$ & $2 \cdot 1$ & $125 \cdot 7$ & $2 \cdot 0$ & $127 \cdot 3$ & $2 \cdot 1$ & $127 \cdot 1$ & 1.6 & \\
\hline Wo & $126 \cdot 1$ & $1 \cdot 6$ & $125 \cdot 1$ & 1.4 & $124 \cdot 8$ & 1.5 & $126 \cdot 6$ & 1.5 & \\
\hline \multicolumn{10}{|l|}{ Diastolic } \\
\hline Control & $75 \cdot 6$ & 1.6 & 71.5 & 1.4 & 74.6 & 1.4 & $72 \cdot 1$ & $1 \cdot 4$ & NS \\
\hline OJ & 74.9 & 1.5 & $70 \cdot 1$ & $1 \cdot 2$ & 73.7 & 1.5 & 70.4 & 1.5 & \\
\hline FROJ & $75 \cdot 5$ & 1.5 & $70 \cdot 9$ & $1 \cdot 6$ & 74.6 & 1.5 & $72 \cdot 2$ & 1.4 & \\
\hline WO & $76 \cdot 0$ & 1.6 & 69.9 & 1.4 & 73.9 & 1.5 & 70.9 & 1.5 & \\
\hline
\end{tabular}
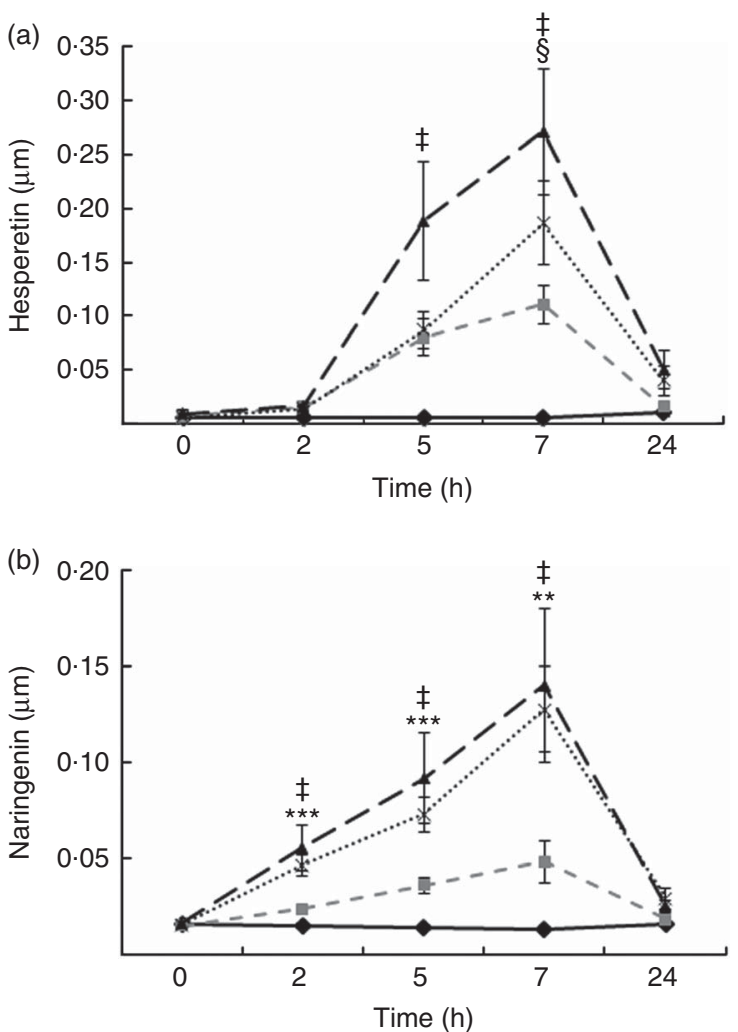

Fig. 3. Plasma flavanone profile following postprandial consumption of flavanone beverages containing either $128.88 \mathrm{mg}$ of flavanones (OJ, - - $-272.14 \mathrm{mg}$ of flavanones (FROJ, - - 4 ), $452.80 \mathrm{mg}$ of flavanones (WO, .......) or a macronutrient- and micronutrient-matched control (—) in middle-aged healthy men $(n$ 20). (a) Hesperetin, (b) naringenin. Values are means, with their standard errors and analysed using a two-factor repeated-measures ANOVA with time and treatment as the two factors (significant main effects of time $\times$ treatment $(P<0.0001)$, time $(P<0.0001)$ and treatment $(P<0.001)$. Post hoc analyses were conducted using Bonferroni's multiple comparison test. Hesperetin levels are significantly higher in all treatments in comparison with control at 5 and $7 \mathrm{~h}(\ddagger 0.00<P<0.02)$, whereas naringenin levels are significantly higher in all treatments in comparison with control at 2,5 and $7 \mathrm{~h}(\ddagger 0.00<P<0.03)$. Levels of plasma naringenin are significantly higher in $\mathrm{OJ}$ in comparison with WO: ${ }^{\star \star} P<0.01$, ${ }^{\star \star \star} P<0.001$. $\S$ Levels of plasma hesperetin in FROJ and WO show a trend towards higher values than OJ $(P<0.1)$. OJ, orange juice; FROJ, flavanone-rich orange juice; WO, whole blended orange.

accurately endogenous NO production in humans (estimate of $70-80 \%$ of plasma nitrite deriving from eNOS activity), whereas the other major source is diet-derived nitrate (by reduction to nitrite). This was the rational for detecting separately levels of nitrite, nitrate and other RXNO species. The average levels of plasma nitrate, nitrite and other RXNO at baseline were $32 \cdot 1 \mu \mathrm{mol} / 1,68.3 \mathrm{nmol} / 1$ and $0.4 \mathrm{nmol} / 1$, respectively, which is in agreement with the values reported in the literature ${ }^{(37,54)}$. A significant decrease in plasma nitrate levels was observed at 2,5 and $7 \mathrm{~h}$ for all the interventions, including control $(P<0.0001)$ (Fig. 4(b)). In contrast, plasma nitrite levels remained constant up to $7 \mathrm{~h}$ after treatment (not significantly different from baseline) following OJ, FROJ and WO intake, whereas the control group nitrite levels decreased significantly $(P<0.01)$ (Fig. 4(a)). No significant changes were detected in RXNO levels in plasma (NS) (Fig. 4(c)).

\section{Discussion}

Considerable evidence suggests that dysregulation of endothelial function in the postprandial state is an important contributing factor for CVD $\operatorname{risk}^{(2,5,7,8)}$, whereas intakes of flavonoid/ polyphenol-rich foods such as cocoa, tea and berries have been shown to exert positive effects on vascular function. In support of this, clinical trial data have indicated that intakes of such foods/beverages may lower CVD disease risk, at least partially, through their actions in mitigating fed-state metabolic and vascular disturbances ${ }^{(34)}$. In the present study, we showed that intervention with orange flavanones, both as juice and whole orange in homogenised form, counteracts impairments in vascular function evoked by a sequential double-meal challenge, which reflects a regular eating pattern and a typical dietary intake in the population ${ }^{(48)}$. Each flavanone intervention tested was effective in reversing vascular impairments, to a physiologically similar degree, despite them containing different levels of flavanones (ranging from 128 to $452 \mathrm{mg}$ ) and resulting in different concentrations of plasma flavanone metabolites. No changes in blood pressure were observed. The rescue of transient impairments in vascular function, as assessed using brachial artery FMD, coincided with the peak of flavanone metabolites (total sulphates and glucuronides) in circulation $(7 \mathrm{~h}$ ) and with sustained levels of plasma nitrite, the latter of which was significantly reduced by the double-meal challenge. Thus, our data support the concept that the observed postprandial vascular benefits may be linked to the actions of circulating flavanone metabolites on NO bioavailability. 

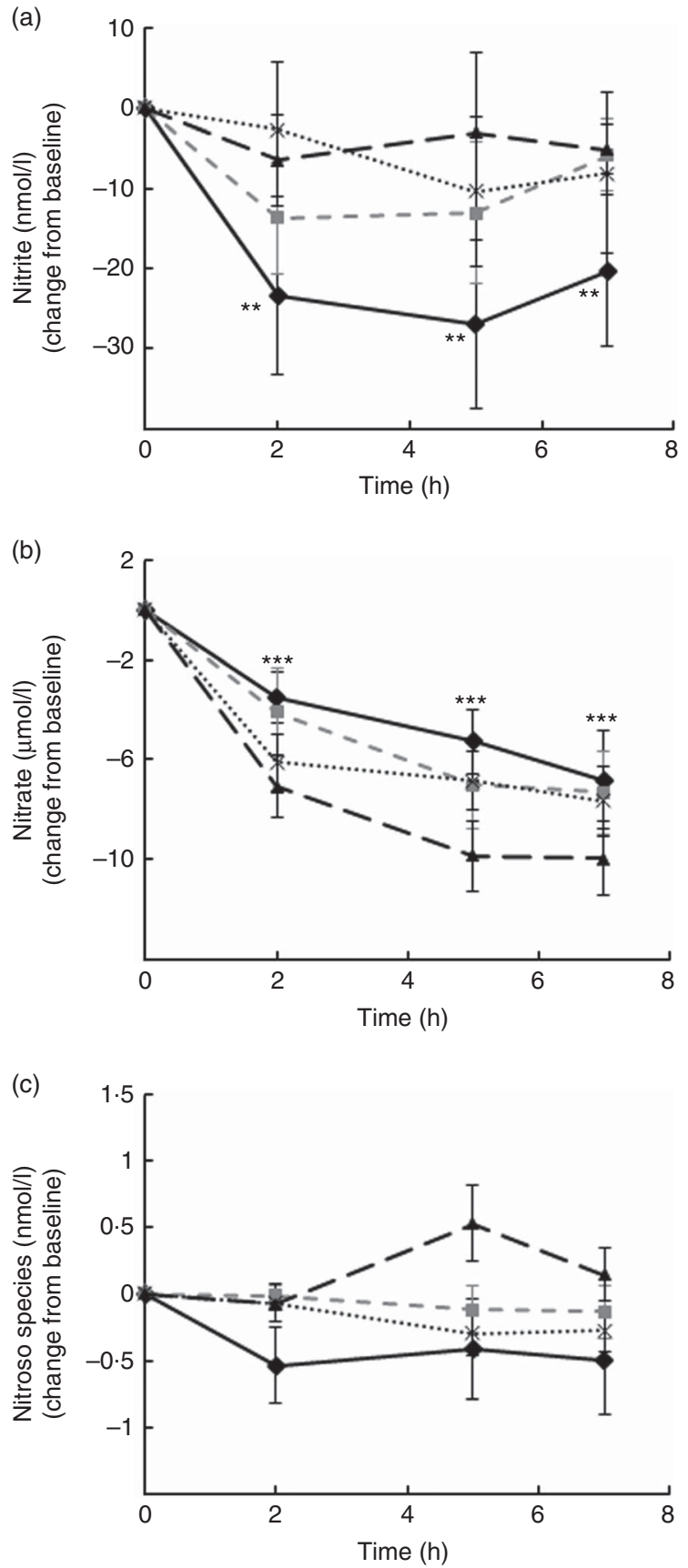

Fig. 4. Plasma nitric oxide levels following postprandial consumption of flavanone beverages containing either $128.88 \mathrm{mg}$ of flavanones (OJ, - 다-), $272.14 \mathrm{mg}$ of flavanones (FROJ, - - -), $452.80 \mathrm{mg}$ of flavanones (WO, .......) or a macronutrient- and micronutrient-matched control ( - ) in middle-aged healthy men ( $n$ 28). (a) Nitrite levels ( $\mathrm{nmol} / \mathrm{l})$, (b) nitrate levels ( $\mu \mathrm{mol} / \mathrm{l})$, (c) nitroso species including nitrosothiols, nitrosamines, iron-nitrosylhaemoglobin and nitrosohaemoglobin $(\mathrm{nmol} / \mathrm{l})$. Values are means, with their standard errors and expressed as change from baseline. Data were analysed using a two-factor repeated-measures ANOVA with time and treatment as the two factors (significant main effects of time $(P<0.0001))$. Post hoc analyses were conducted using Bonferroni's multiple comparison test. ${ }^{* *}$ Nitrite levels are significantly different from baseline only for the control group at the specified time points $(P<0.01)$. ${ }^{* * *}$ Nitrate levels are significantly different from baseline for both control and all three flavanone treatments $(P<0.0001)$ at the specified time points. OJ, orange juice; FROJ, flavanone-rich orange juice; WO, whole blended orange. 
Our findings are consistent with previous randomised controlled trial (RCT) data sets indicating that cocoa flavanols partially counteract the decrease in FMD following high-fat meal loading ${ }^{(32)}$. Furthermore, pure quercetin has also been shown to ameliorate postprandial FMD following maltose overload $^{(33)}$. To our knowledge, this is the first set of data indicating that citrus flavanones are also capable of attenuating postprandial impairments in endothelial function following a sequential high-medium-fat double meal in individuals displaying mild cardiometabolic risk factors. Furthermore, and in support of our findings, previous studies conducted in the fasted state report that chronic interventions with flavanones in at-risk groups (e.g. hypertensive, overweight or the metabolic syndrome patients) induce positive effects on blood pressure and endothelial function (FMD) ${ }^{(45,46,55)}$ and improvements in microvascular reactivity ${ }^{(45)}$. Most importantly, in the present study, no dose-dependent effects on brachial artery FMD were observed, despite the interventions containing different amounts of flavanones (WO: $3 \cdot 5 \times \mathrm{OJ}$ ). This may indicate that at these intake levels a threshold plateau may be reached, similar to what has been shown previously with other flavonoid-rich interventions $^{(22)}$. It also further suggests that lower doses of flavanones (approximately $130 \mathrm{mg}$ ) can be efficacious at modulating postprandial endothelial function. No changes in blood pressure were observed in the present study, which is in agreement with previous human intervention trials reporting modulation of blood pressure only after chronic interventions with flavonoid-rich foods, but not in an acute manner ${ }^{(27,31)}$

We observed concurrent modulation of FMD, nitrite and circulating flavanone metabolites (total sulphates and glucuronides), suggesting that the latter may be linked to NO availability and subsequent improvements in vascular function, although we cannot establish a causal relationship at this time. Specifically, both naringenin and hesperetin metabolite peak plasma levels for all three interventions occured at approximately $7 \mathrm{~h}$, which coincided with significant improvements in endothelial function (at $7 \mathrm{~h}$ ) and sustained levels of circulating nitrite after flavanone interventions in comparison with control. Plasma levels of flavanone metabolites peaked slightly later than that previously reported $(4-6 \mathrm{~h})^{(45,56,57)}$, most likely due to the concomitant intake of fat, which is thought to interfere with flavonoid absorption ${ }^{(58)}$. On the other hand, no significant differences in time of absorption were detected between the flavanone treatment groups (peak occurs at approximately $7 \mathrm{~h}$ for all three treatments).

In support of the link between flavanone intake and human vascular function, we observed that when low or no levels of flavanone metabolites are detected in circulation (e.g. 2 h), no differences in postprandial brachial FMD are observed between control and flavanone-rich beverages. Further, multiple regression analyses suggest that mainly hesperetin metabolites seem to predict significantly the magnitude of changes in FMD ( $r 0.32, P=0.005$ ), suggesting an important role of this flavanone in the effects observed. This is corroborated by previous studies showing that pure hesperidin can trigger both acute and chronic improvements in vascular function in humans ${ }^{(45,46)}$. It is important to further note that only the sulphated and glucuronidated portion of the flavanone metabolites were quantified in our study, and these are likely to account for a fraction (approximately 16\%) of the total flavanone metabolites absorbed $^{(59)}$. As such, we anticipate that gut-derived phenolic compounds might also contribute to the improvements in endothelial function observed. This is supported by our observation that hesperetin metabolites can only significantly predict a small percentage (approximately $30 \%$ ) of the FMD response observed; therefore, it is likely that stronger correlations might be apparent once gut-derived small phenolic metabolites are taken into consideration; however, such extensive analysis was outside of the scope of our study.

Our study also indicates that the impairment in postprandial FMD induced by the sequential high-fat meal might be linked to decreases in circulating levels of NO species, particularly nitrite and nitrate. Although the precise mechanisms underlying postprandial endothelium impairments are not established, mechanistic animal studies suggest a role for NO signalling, showing, for example, that endothelial dysfunction induced by fat intake also results in decreases in NO production ${ }^{(60-62)}$. Importantly, the flavanone interventions only prevented the decrease in nitrite, but not nitrate. Numerous evidence suggest that nitrite reflects more accurately endogenous NO production in humans, with an estimate of $70-80 \%$ of plasma nitrite deriving from eNOS activity ${ }^{(63,64)}$ and also better reflects the degree of endothelial dysfunction in humans ${ }^{(65)}$. In agreement with our data, previous human clinical data suggest an ability of some flavonoid-rich foods to modulate NO bioavailability ${ }^{(22,24,35,37)}$. In particular, cocoa flavanols-induced improvements in FMD have been causally linked to NO production in humans ${ }^{(23)}$. More recently, Bondonno et $a l{ }^{(37)}$, also showed that apples containing (-) epicatechin and quercetin increased levels of nitrite along with FMD response after $2 \mathrm{~h}$ of intake. In addition, and in agreement with the present data, both pure (-) epicatechin and quercetin were shown to specifically increase plasma nitrite, but not nitrate in healthy humans ${ }^{(35)}$. Supporting in vitro mechanistic studies (in endothelial cells) have demonstrated that flavanone hesperetin and some of its in vivo metabolites (e.g. $7-O-\beta$-D glucuronide) can stimulate $\mathrm{NO}$ production via activation/ expression of $\operatorname{eNOS}^{(46,66)}$ or by decreasing NO degradation through inhibition of $\mathrm{NADPH}^{(67)}$, and these are possible mechanistic pathways by which flavanone metabolites might modulate postprandial FMD. Although the specific modulation of nitrite by flavanone-containing interventions is an interesting observation in the present study, the interpretation of the temporal dynamics (time course) of flavanone appearance in plasma and levels of plasma nitrite is not straightforward with regard to explaining the effects of nitrite on FMD. This seems to suggest that the impact of flavanone metabolites on FMD response cannot be explained completely by modulation of nitrite (as a measure of $\mathrm{NO}$ ). As the present study was not designed (or powered) to detect changes in NO species, we are limited in our ability to establish a clear-cut link between FMD modulation and NO at this time. However, we believe this preliminary data are very novel and valuable for future, more mechanism-focused, human RCT.

Interestingly, FROJ intake resulted in similar levels of plasma flavanones to WO, despite lower initial concentrations, which might be related to characteristics of the food matrix itself, such as viscosity, which is known to influence the bioavailability of 
polyphenols $^{(68)}$. It is possible that the reduced particle size of pomace in FROJ aided the release of polyphenols from the fibre matrix, making these more accessible for gut microbiota metabolism $^{(69)}$. It is known that dietary fibre can physically trap polyphenols within the fibre matrix in the fruit tissue, reducing the accessibility to enzymes and the gut microbiota ${ }^{(70)}$. On the other hand, the rate of release of polyphenols from fibrous particles is inversely proportional to the fibre particle size ${ }^{(71)}$; therefore, by reducing particle size in the pomace, we are likely to increase the bioavailability of flavanones in FROJ. In order to confirm that this is the case, future studies should focus on measuring accurately total urine excretion (e.g. over a $24 \mathrm{~h}$ period). Nonetheless, our study seems to suggest that particle size reduction of fibre-rich orange pomace and the re-introduction of this product into orange juice might be an effective strategy to increase the bioavailability of polyphenols in vivo. Importantly, the increased bioavailability of flavanones after FROJ intake did not enhance significantly postprandial FMD in comparison with lower flavanone-containing OJ, again suggesting that perhaps a certain level of flavanone metabolites is necessary in circulation to trigger postprandial FMD improvements, but further increases in flavanone levels may not produce additional benefits ${ }^{(22)}$.

One of the limitations in the design of the present study is related to the composition of the control intervention, which did not take into account the levels of ascorbic acid present in the citrus beverages. Clinical studies suggest that doses up to $500 \mathrm{mg}$ of vitamin $\mathrm{C}$ do not impact on biomarkers of endothelial function $^{(49,72)}$. More specifically, it has been shown in a recent stratified meta-analysis that doses ranging from 90 to $500 \mathrm{mg}$ of ascorbic acid do not produce improvements in endothelial function, both acutely or chronically ${ }^{(73,74)}$, especially in populations with normal vitamin $\mathrm{C}$ status ${ }^{(75,76)}$. Furthermore, previous studies reporting acute beneficial effects of ascorbic acid on endothelium-dependent vasodilation (within 2-4h of intake) delivered doses of at least $2000 \mathrm{mg}^{(77-79)}$, and in many cases positive outcomes were achieved by delivering ascorbic acid intravenously, resulting in supra-physiological plasma levels of vitamin $\mathrm{C}$, which cannot be achieved by oral ingestion ${ }^{(79,80)}$. As the levels of vitamin $\mathrm{C}$ in the present study were approximately 80-120 mg, we are confident that these can be considered negligible with respect to acute effects on endothelial function, as measured by FMD. Therefore, despite these limitations, we can safely argue that our conclusions are reasonable when attributing the FMD response to circulating flavanone metabolites (at $7 \mathrm{~h}$ post intake) and that our data are relevant in furthering the understanding of the impact of flavonoid-rich foods/beverages on postprandial endothelial function.

In summary, our results suggest that acute intake of a beverage containing at least $128 \mathrm{mg}$ of flavanones can be an effective dietary strategy to blunt the acute transient impairment in endothelial function induced by a sequential double meal that reflects a typical intake in the population. Although we cannot draw firm conclusions regarding the mechanisms by which flavanones elicit vascular responses, our results suggest that these might be linked to an ability of flavanone metabolites to sustain basal circulating NO levels. Collectively, these observations have important implications, considering that most individuals spend the majority of the day in the postprandial state, and such temporary vascular changes repeated on a daily basis can critically impact on long-term vascular health and overall chronic disease risk.

\section{Acknowledgements}

This work was supported by PepsiCo Inc. (PEP-1122) and is greatly appreciated. The views expressed in this manuscript are those of the authors and do not necessarily reflect the position or policy of PepsiCo Inc.

C. R.: coordinated and conducted the study, undertook all FMD measurements, performed data analysis and wrote the manuscript; H. D.: coordinated and conducted the study; C. S.: designed and coordinated the study and wrote the manuscript; L. H.: designed the study; R. L. B.: conducted study drinks analysis; M. B., Y. H.: conducted plasma flavanone analysis; G. C.: conducted NO species measurements and analysis; J. A. L.: co-investigated the study and was involved in experimental design; J. P. E. S.: principal investigator, involved in the experimental design and writing of the manuscript. All authors reviewed the manuscript.

C. S. works as a Senior Scientist at PepsiCo Inc., L. H. works as a Senior Director at Global R\&D Nutrition at PepsiCo Inc., R. L. B., M. B. and Y. H. work as Principle Scientists at PepsiCo Inc. The other authors declare no conflicts of interest.

\section{References}

1. Ceriello A, Taboga C, Tonutti L, et al. (2002) Evidence for an independent and cumulative effect of postprandial hypertriglyceridemia and hyperglycemia on endothelial dysfunction and oxidative stress generation: effects of short- and long-term simvastatin treatment. Circulation 106, 1211-1218.

2. de Koning EJ \& Rabelink TJ (2002) Endothelial function in the post-prandial state. Atheroscler Suppl 3, 11-16.

3. Nappo F, Esposito K, Cioffi M, et al. (2002) Postprandial endothelial activation in healthy subjects and in type 2 diabetic patients: role of fat and carbohydrate meals. J Am Coll Cardiol 39, $1145-1150$.

4. Alipour A, Elte JW, van Zaanen HC, et al. (2007) Postprandial inflammation and endothelial dysfuction. Biochem Soc Trans 35, 466-469.

5. Ebenbichler CF, Kirchmair R, Egger C, et al. (1995) Postprandial state and atherosclerosis. Curr Opin Lipidol 6, 286-290.

6. Karpe F (1997) Postprandial lipid metabolism in relation to coronary heart disease. Proc Nutr Soc 56, 671-678.

7. Ansar S, Koska J \& Reaven PD (2011) Postprandial hyperlipidemia, endothelial dysfunction and cardiovascular risk: focus on incretins. Cardiovasc Diabetol 10, 61.

8. Ceriello A (2000) The post-prandial state and cardiovascular disease: relevance to diabetes mellitus. Diabetes Metab Res Rev 16, 125-132.

9. Newens KJ, Thompson AK, Jackson KG, et al. (2011) DHA-rich fish oil reverses the detrimental effects of saturated fatty acids on postprandial vascular reactivity. Am J Clin Nutr 94, 742-748.

10. Jackson KG, Armah CK \& Minihane AM (2007) Meal fatty acids and postprandial vascular reactivity. Biochem Soc Trans $\mathbf{3 5}$, 451-453.

11. Vogel RA, Corretti MC \& Plotnick GD (1997) Effect of a single high-fat meal on endothelial function in healthy subjects. $A m J$ Cardiol 79, 350-354. 
12. Keogh JB, Grieger JA, Noakes M, et al. (2005) Flow-mediated dilatation is impaired by a high-saturated fat diet but not by a high-carbohydrate diet. Arterioscler Thromb Vascu Biol 25, 1274-1279.

13. Steer P, Sarabi DM, Karlstrom B, et al. (2003) The effect of a mixed meal on endothelium-dependent vasodilation is dependent on fat content in healthy humans. Clin Sci (Lond) 105, 81-87.

14. Steinberg HO, Tarshoby M, Monestel R, et al. (1997) Elevated circulating free fatty acid levels impair endotheliumdependent vasodilation. J Clin Invest 100, 1230-1239.

15. Bae JH, Bassenge E, Kim KB, et al. (2001) Postprandial hypertriglyceridemia impairs endothelial function by enhanced oxidant stress. Atherosclerosis 155, 517-523.

16. Cassidy A, Mukamal KJ, Liu L, et al. (2013) High anthocyanin intake is associated with a reduced risk of myocardial infarction in young and middle-aged women. Circulation 127, 188-196.

17. Mink PJ, Scrafford CG, Barraj LM, et al. (2007) Flavonoid intake and cardiovascular disease mortality: a prospective study in postmenopausal women. Am J Clin Nutr 85, 895-909.

18. McCullough ML, Peterson JJ, Patel R, et al. (2012) Flavonoid intake and cardiovascular disease mortality in a prospective cohort of US adults. Am J Clin Nutr 95, 454-464.

19. Zamora-Ros R, Jimenez C, Cleries R, et al. (2013) Dietary flavonoid and lignan intake and mortality in a Spanish cohort. Epidemiology 24, 726-733.

20. Wang X, Ouyang YY, Liu J, et al. (2014) Flavonoid intake and risk of CVD: a systematic review and meta-analysis of prospective cohort studies. Br J Nutr 111, 1-11.

21. Rodriguez-Mateos A, Del Pino-Garcia R, George TW, et al. (2014) Impact of processing on the bioavailability and vascular effects of blueberry (poly)phenols. Mol Nutr Food Res 58, 1952-1961

22. Rodriguez-Mateos A, Rendeiro C, Bergillos-Meca $\mathrm{T}$, et al. (2013) Intake and time dependence of blueberry flavonoidinduced improvements in vascular function: a randomized, controlled, double-blind, crossover intervention study with mechanistic insights into biological activity. Am J Clin Nutr $\mathbf{9 8}$ 1179-1191.

23. Heiss C, Kleinbongard P, Dejam A, et al. (2005) Acute consumption of flavanol-rich cocoa and the reversal of endothelial dysfunction in smokers. J Am Coll Cardiol 46, 1276-1283.

24. Schroeter H, Heiss C, Balzer J, et al. (2006) (-)-Epicatechin mediates beneficial effects of flavanol-rich cocoa on vascular function in humans. Proc Natl Acad Sci U S A 103, 1024-1029.

25. Fisher ND, Hughes M, Gerhard-Herman M, et al. (2003) Flavanol-rich cocoa induces nitric-oxide-dependent vasodilation in healthy humans. J Hypertens 21, 2281-2286.

26. Heiss C, Finis D, Kleinbongard P, et al. (2007) Sustained increase in flow-mediated dilation after daily intake of high-flavanol cocoa drink over 1 week. J Cardiovasc Pharmacol 49, 74-80.

27. Heiss C, Sansone R, Karimi H, et al. (2015) Impact of cocoa flavanol intake on age-dependent vascular stiffness in healthy men: a randomized, controlled, double-masked trial. Age (Dordr) 37, 9794.

28. Chanet A, Milenkovic D, Manach C, et al. (2012) Citrus flavanones: what is their role in cardiovascular protection? J Agric Food Chem 60, 8809-8822.

29. Hooper L, Kay C, Abdelhamid A, et al. (2012) Effects of chocolate, cocoa, and flavan-3-ols on cardiovascular health: a systematic review and meta-analysis of randomized trials. $\mathrm{Am}$ J Clin Nutr 95, 740-751.

30. Shrime MG, Bauer SR, McDonald AC, et al. (2011) Flavonoid-rich cocoa consumption affects multiple cardiovascular risk factors in a meta-analysis of short-term studies. J Nutr 141, 1982-1988.
31. Sansone R, Rodriguez-Mateos A, Heuel J, et al. (2015) Cocoa flavanol intake improves endothelial function and Framingham Risk Score in healthy men and women: a randomised, controlled, double-masked trial: the Flaviola Health Study. Br J Nutr 114, 1246-1255.

32. Westphal S \& Luley C (2011) Flavanol-rich cocoa ameliorates lipemia-induced endothelial dysfunction. Heart Vessels 26, 511-515.

33. Nakayama H, Tsuge N, Sawada H, et al. (2013) Chronic intake of onion extract containing quercetin improved postprandial endothelial dysfunction in healthy men. J Am Coll Nutr 32, 160-164.

34. Burton-Freeman B (2010) Postprandial metabolic events and fruit-derived phenolics: a review of the science. Br J Nutr 104, Suppl. 3, S1-S14.

35. Loke WM, Hodgson JM, Proudfoot JM, et al. (2008) Pure dietary flavonoids quercetin and (-)-epicatechin augment nitric oxide products and reduce endothelin-1 acutely in healthy men. Am J Clin Nutr 88, 1018-1025.

36. Zhu Y, Xia M, Yang Y, et al. (2011) Purified anthocyanin supplementation improves endothelial function via NO-cGMP activation in hypercholesterolemic individuals. Clin Chem $\mathbf{5 7}$, 1524-1533.

37. Bondonno CP, Yang X, Croft KD, et al. (2012) Flavonoid-rich apples and nitrate-rich spinach augment nitric oxide status and improve endothelial function in healthy men and women: a randomized controlled trial. Free Radic Biol Med 52, $95-102$.

38. Duarte J, Francisco V \& Perez-Vizcaino F (2014) Modulation of nitric oxide by flavonoids. Food Funct 5, 1653-1668.

39. Engler MB, Engler MM, Chen CY, et al. (2004) Flavonoid-rich dark chocolate improves endothelial function and increases plasma epicatechin concentrations in healthy adults. J Am Coll Nutr 23, 197-204.

40. Monahan KD, Feehan RP, Kunselman AR, et al. (2011) Dosedependent increases in flow-mediated dilation following acute cocoa ingestion in healthy older adults. $J$ Appl Physiol 111, 1568-1574.

41. Heiss C, Jahn S, Taylor M, et al. (2010) Improvement of endothelial function with dietary flavanols is associated with mobilization of circulating angiogenic cells in patients with coronary artery disease. J Am Coll Cardiol 56, 218-224.

42. Heiss C, Dejam A, Kleinbongard P, et al. (2003) Vascular effects of cocoa rich in flavan-3-ols. JAMA 290, 1030-1031.

43. Balzer J, Rassaf T, Heiss C, et al. (2008) Sustained benefits in vascular function through flavanol-containing cocoa in medicated diabetic patients a double-masked, randomized, controlled trial. J Am Coll Cardiol 51, 2141-2149.

44. Del Rio D, Rodriguez-Mateos A, Spencer JP, et al. (2013) Dietary (poly)phenolics in human health: structures, bioavailability, and evidence of protective effects against chronic diseases. Antioxid Redox Signal 18, 1818-1892.

45. Morand C, Dubray C, Milenkovic D, et al. (2011) Hesperidin contributes to the vascular protective effects of orange juice: a randomized crossover study in healthy volunteers. Am J Clin Nutr 93, 73-80.

46. Rizza S, Muniyappa R, Iantorno M, et al. (2011) Citrus polyphenol hesperidin stimulates production of nitric oxide in endothelial cells while improving endothelial function and reducing inflammatory markers in patients with metabolic syndrome. J Clin Endocrinol Metab 96, E782-E792.

47. Santamaria P (2006) Nitrate in vegetables: toxicity, content, intake and EC regulation. $J$ Sci Food Agric 86, 10-17.

48. Jackson KG, Clarke DT, Murray P, et al. (2010) Introduction to the DISRUPT postprandial database: subjects, studies and methodologies. Genes Nutr 5, 39-48. 
49. Seljeflot I, Arnesen H, Brude IR, et al. (1998) Effects of omega-3 fatty acids and/or antioxidants on endothelial cell markers. Eur J Clin Invest 28, 629-635.

50. McRae MP (2009) High-dose folic acid supplementation effects on endothelial function and blood pressure in hypertensive patients: a meta-analysis of randomized controlled clinical trials. J Chiropr Med 8, 15-24

51. Corretti MC, Anderson TJ, Benjamin EJ, et al. (2002) Guidelines for the ultrasound assessment of endothelial-dependent flow-mediated vasodilation of the brachial artery: a report of the International Brachial Artery Reactivity Task Force. I Am Coll Cardiol 39, 257-265.

52. Matsumoto H, Ikoma Y, Sugiura M, et al. (2004) Identification and quantification of the conjugated metabolites derived from orally administered hesperidin in rat plasma. J Agric Food Chem 52, 6653-6659.

53. Ignarro LJ, Fukuto JM, Griscavage JM, et al. (1993) Oxidation of nitric oxide in aqueous solution to nitrite but not nitrate: comparison with enzymatically formed nitric oxide from L-arginine. Proc Natl Acad Sci U S A 90, 8103-8107.

54. Rassaf T, Heiss C, Hendgen-Cotta U, et al. (2006) Plasma nitrite reserve and endothelial function in the human forearm circulation. Free Radic Biol Med 41, 295-301.

55. Reshef N, Hayari Y, Goren C, et al. (2005) Antihypertensive effect of sweetie fruit in patients with stage I hypertension. $A m$ J Hypertens 18, 1360-1363.

56. Mullen W, Archeveque MA, Edwards CA, et al. (2008) Bioavailability and metabolism of orange juice flavanones in humans: impact of a full-fat yogurt. J Agric Food Chem 56, $11157-11164$

57. Erlund I, Meririnne E, Alfthan G, et al. (2001) Plasma kinetics and urinary excretion of the flavanones naringenin and hesperetin in humans after ingestion of orange juice and grapefruit juice. J Nutr 131, 235-241.

58. Mullen W, Edwards CA, Serafini M, et al. (2008) Bioavailability of pelargonidin-3-O-glucoside and its metabolites in humans following the ingestion of strawberries with and without cream. J Agric Food Chem 56, 713-719.

59. Pereira-Caro G, Borges G, van der Hooft J, et al. (2014) Orange juice (poly)phenols are highly bioavailable in humans. Am J Clin Nutr 100, 1378-1384.

60. Magne J, Huneau JF, Delemasure S, et al. (2009) Whole-body basal nitric oxide production is impaired in postprandial endothelial dysfunction in healthy rats. Nitric Oxide 21, 37-43.

61. Erdei N, Toth A, Pasztor ET, et al. (2006) High-fat diet-induced reduction in nitric oxide-dependent arteriolar dilation in rats: role of xanthine oxidase-derived superoxide anion. $A m J$ Physiol Heart Circ Physiol 291, H2107-H2115.

62. Razny U, Kiec-Wilk B, Wator L, et al. (2011) Increased nitric oxide availability attenuates high fat diet metabolic alterations and gene expression associated with insulin resistance. Cardiovasc Diabetol 10, 68.

63. Lauer T, Preik M, Rassaf T, et al. (2001) Plasma nitrite rather than nitrate reflects regional endothelial nitric oxide synthase activity but lacks intrinsic vasodilator action. Proc Natl Acad Sci U S A 98, 12814-12819.

64. Kleinbongard P, Dejam A, Lauer T, et al. (2003) Plasma nitrite reflects constitutive nitric oxide synthase activity in mammals. Free Radic Biol Med 35, 790-796.
65. Kleinbongard P, Dejam A, Lauer T, et al. (2006) Plasma nitrite concentrations reflect the degree of endothelial dysfunction in humans. Free Radic Biol Med 40, 295-302.

66. Liu L, Xu DM \& Cheng YY (2008) Distinct effects of naringenin and hesperetin on nitric oxide production from endothelial cells. J Agric Food Chem 56, 824-829.

67. Takumi H, Nakamura H, Simizu T, et al. (2012) Bioavailability of orally administered water-dispersible hesperetin and its effect on peripheral vasodilatation in human subjects: implication of endothelial functions of plasma conjugated metabolites. Food Funct 3, 389-398.

68. Bohn T (2014) Dietary factors affecting polyphenol bioavailability. Nutr Rev 72, 429-452.

69. Palafox-Carlos H, Ayala-Zavala JF \& Gonzalez-Aguilar GA (2011) The role of dietary fiber in the bioaccessibility and bioavailability of fruit and vegetable antioxidants. J Food Sci 76. R6-R15.

70. Montagne L, Pluske JR \& Hampson DJ (2003) A review of interactions between dietary fibre and the intestinal mucosa, and their consequences on digestive health in young nonruminant animals. Anim Feed Sci Technol 108, 95-117.

71. Cummings JH, Edmond LM \& Magee EA (2004) Dietary carbohydrates and health: do we still need the concept of fibre? Clin Nutr Suppl 1, 5-17.

72. Ward NC, Hodgson JM, Croft KD, et al. (2005) The combination of vitamin $\mathrm{C}$ and grape-seed polyphenols increases blood pressure: a randomized, double-blind, placebocontrolled trial. J Hypertens 23, 427-434.

73. Ashor AW, Lara J, Mathers JC, et al. (2014) Effect of vitamin C on endothelial function in health and disease: a systematic review and meta-analysis of randomised controlled trials. Atherosclerosis 235, 9-20.

74. Ashor AW, Siervo M, Lara J, et al. (2015) Effect of vitamin C and vitamin $\mathrm{E}$ supplementation on endothelial function: a systematic review and meta-analysis of randomised controlled trials. Br J Nutr 113, 1182-1194.

75. Frikke-Schmidt H \& Lykkesfeldt J (2009) Role of marginal vitamin $\mathrm{C}$ deficiency in atherogenesis: in vivo models and clinical studies. Basic Clin Pharmacol Toxicol 104, 419-433.

76. Harris RA, Nishiyama SK, Wray DW, et al. (2009) The effect of oral antioxidants on brachial artery flow-mediated dilation following 5 and 10 min of ischemia. Eur J Appl Physiol 107, 445-453.

77. Raitakari OT, Adams MR, McCredie RJ, et al. (2000) Oral vitamin $\mathrm{C}$ and endothelial function in smokers: short-term improvement, but no sustained beneficial effect. $J$ Am Coll Cardiol 35, 1616-1621.

78. Pullin CH, Bonham JR, McDowell IF, et al. (2002) Vitamin C therapy ameliorates vascular endothelial dysfunction in treated patients with homocystinuria. J Inhert Metab Dis $\mathbf{2 5}$, 107-118.

79. Eskurza I, Monahan KD, Robinson JA, et al. (2004) Effect of acute and chronic ascorbic acid on flow-mediated dilatation with sedentary and physically active human ageing. J Physiol 556, 315-324.

80. Taddei S, Virdis A, Ghiadoni L, et al. (1998) Vitamin C improves endothelium-dependent vasodilation by restoring nitric oxide activity in essential hypertension. Circulation $\mathbf{9 7}$, 2222-2229. 